\title{
Local Group Dynamics
}

\author{
D. Lynden-Bell
}

Institute of Astronomy, University of Cambridge, Madingley Road, Cambridge. CB3 $0 H A U K$

PPARC Senior Fellow on leave at SAAO (September 1998) and M.I.T. (October - December 1998)

\begin{abstract}
The distance from the Local Group to the 'sphere' of small galaxies that no longer expand with the Universe determines the time since the Big Bang, $t \times M^{1 / 2}$, where $M$ is the mass of the Local Group. Adopting Feast's new distance scale, this distance is found to be $1.35 \pm 0.1 \mathrm{Mpc}$. The velocity of approach and the distance to M31 give a different combination of $t$ and $M$, thus both can be deduced. We find the time since the Big Bang
\end{abstract}

$$
t=1.2_{-3}^{+1} \times 10^{10} \mathrm{yr} .
$$

and

$$
M=4.9 \pm .810^{12} \mathrm{M}_{\odot} .
$$

The importance of accurate distances for such results is stressed. If all distances are revised by a factor $\lambda$ then both $t$ and $M$ change by that factor.

\section{Introduction}

To apply dynamics usefully we need to know where most of the mass is, since that provides the gravity. If we look for guidance to the big clusters of galaxies, we find that most of the mass is invisible and in some unknown form which almost certainly is not made of baryons. Most of the directly detected mass is intergalactic hot gas which emits X-rays. This is normally 3 to 5 times the mass of all the stars in the galaxies of the cluster. By contrast the assessments of the baryon content of the Local Group are topsy turvy because these estimates of the total gas mass are $1 / 3$ to $1 / 5$ of the mass in the stars. Either we need to produce a good reason why much more of the gas has been turned into stars in small galaxy groups such as ours or we should be looking for much more intergalactic gas. However, we shall find that the mass of the Local Group is predominantly dark (invisible) matter so in this respect the Local Group mimics the great clusters.

To decide where most of the mass is, we rely on the small fraction that is in stars to give us guidance. 


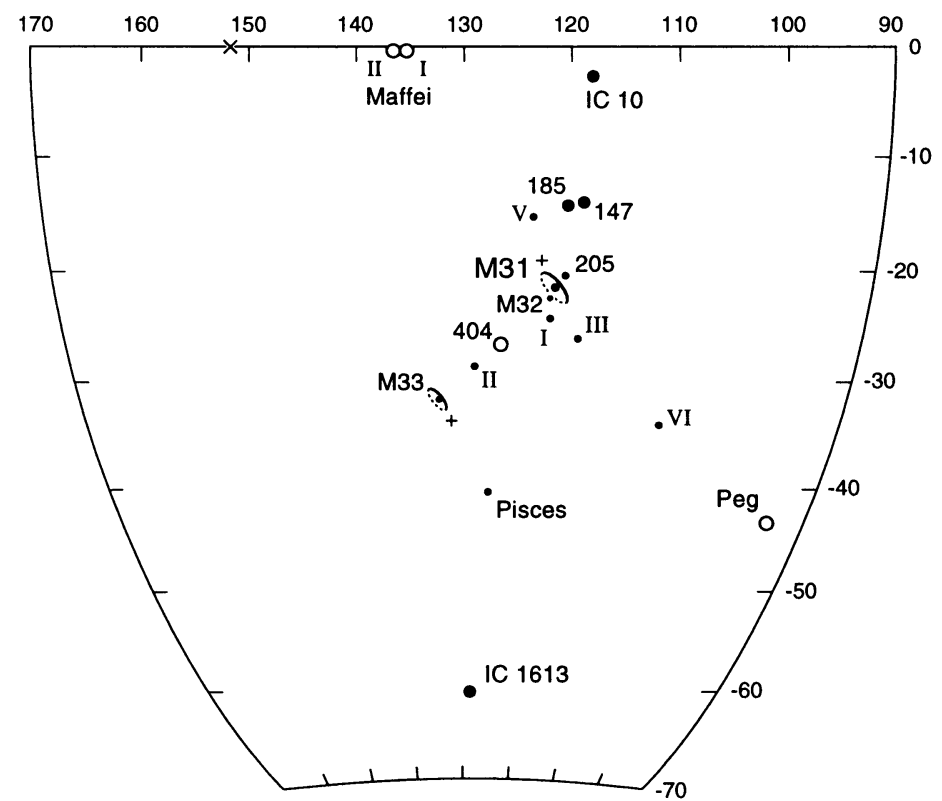

Figure 1. The sky in the direction of Andromeda. The objects represented by open circles are nearby but beyond the Local Group (e.g., Maffei I \& II and the spiral NGC 404). For M31 and M33, + indicates the end rotating away from us and the hard lines indicate the side towards us. The numbers are NGC numbers; I, II, III, V, VI are the dwarf spheroidal companions in the Andromeda subgroup.

\section{The Andromeda Subgroup}

Figure 1 shows the sky in the direction of Andromeda, M31. Marked on it are the Local Galaxies near that direction. There is clearly a strong concentration of them about the dominant member M31. This is evidence that the gravitational potential of the dark matter which keeps the Andromeda subgroup together must be centred on or near to M31. All these galaxies, with the exceptions of NGC 404 and Maffei I \& II at the very top which are chance projections of background galaxies, lie at distances within $30 \%$ of M31. Distances in astronomy have been notoriously bad and the Local Group distances are no exception. Pisces = LGSIII, IC 10, and Pegasus (irreg) 三 DDO 216 were until a few years ago placed at around twice the distance to M31, but better distances now place them quite near to it. It is to be expected that a truly associated galaxy seen at a projected distance $R$ will have a true distance from Andromeda of the order of $\sqrt{3 / 2} R$ because the square of the depth difference should not be radically different from the squares of the two coordinates in the plane of the sky. However, it could be, so that estimate is not always a good one. Figure 2 shows that these new distances make the Andromeda grouping on the sky much more compact in space and that the only other prominent grouping is around the Milky Way. This again is some sort of evidence that the gravity of the dark matter is centred on 


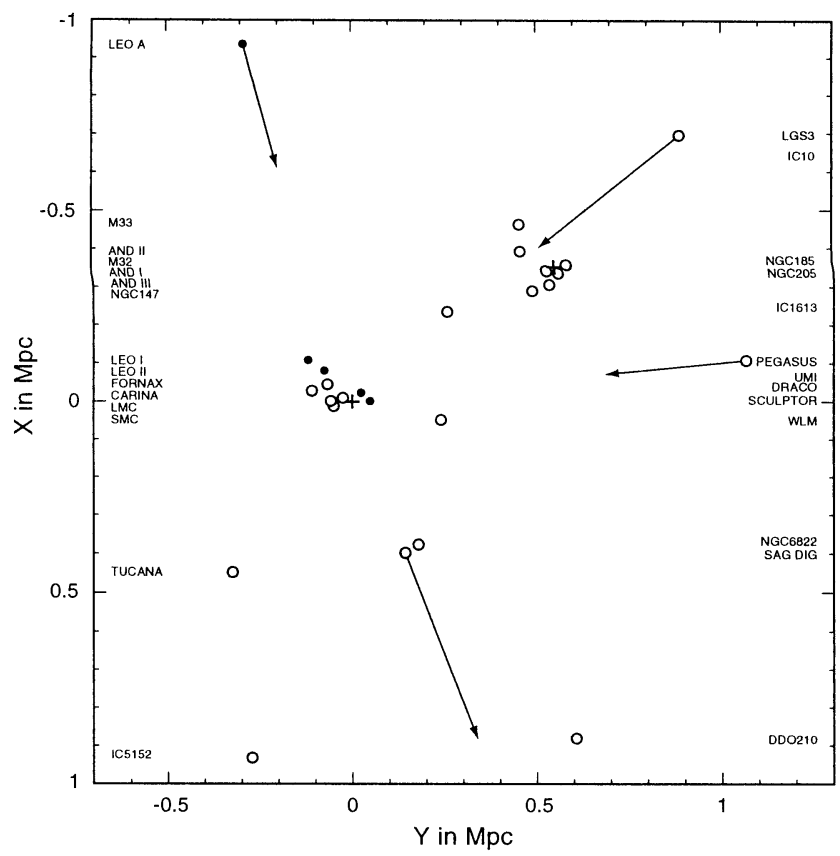

Figure 2. Local Group Galaxies projected on to the Galactic plane.

The arrows indicate recent distance revisions.

the prominent galaxies or at least close to them. Again this follows the giant clusters which often contain a dominant central galaxy about which lies the $\mathrm{X}$-ray emitting gas bound by the cluster's gravity field.

To assess the mass distribution around M31 we can rely on its rotation curve out to about $30 \mathrm{kpc}$ from the centre. Indeed it was M. Roberts' determination of the $21 \mathrm{~cm}$ curve, which remained flat far beyond the optical image, that was one of the early indicators of extended dark matter distributions around galaxies. However, beyond $30 \mathrm{kpc}$ we have to use statistical methods based on the velocities of the satellites. A form of the Virial Theorem appropriate for such data was given by Lynden-Bell \& Frenk (1981). If $\mathbf{r}$ is the radius vector from the centre to an object in orbit and $\psi(\mathbf{r})$ is the gravitational potential in which the object moves then:

$$
\ddot{\mathbf{r}}=\nabla \psi \equiv \frac{\partial \psi}{\partial \mathbf{r}}
$$

hence:

$$
\mathbf{r} \cdot \ddot{\mathbf{r}}=\frac{d^{2}}{d t^{2}}\left(\frac{1}{2} r^{2}\right)-\dot{\mathbf{r}}^{2}=\mathbf{r} \cdot \frac{\partial \psi}{\partial \mathbf{r}}=-V_{c}^{2}
$$

where $V_{c}$ is the transverse velocity whose centrifugal force exactly balances the radial component of gravity at $\mathbf{r}$. Averaging equation (1) over the satellites and assuming that their mean $r^{2}$ is neither accelerating nor decelerating to larger or smaller values, we deduce:

$$
\left\langle\dot{\mathbf{r}}^{2}\right\rangle=\left\langle V_{c}^{2}\right\rangle
$$




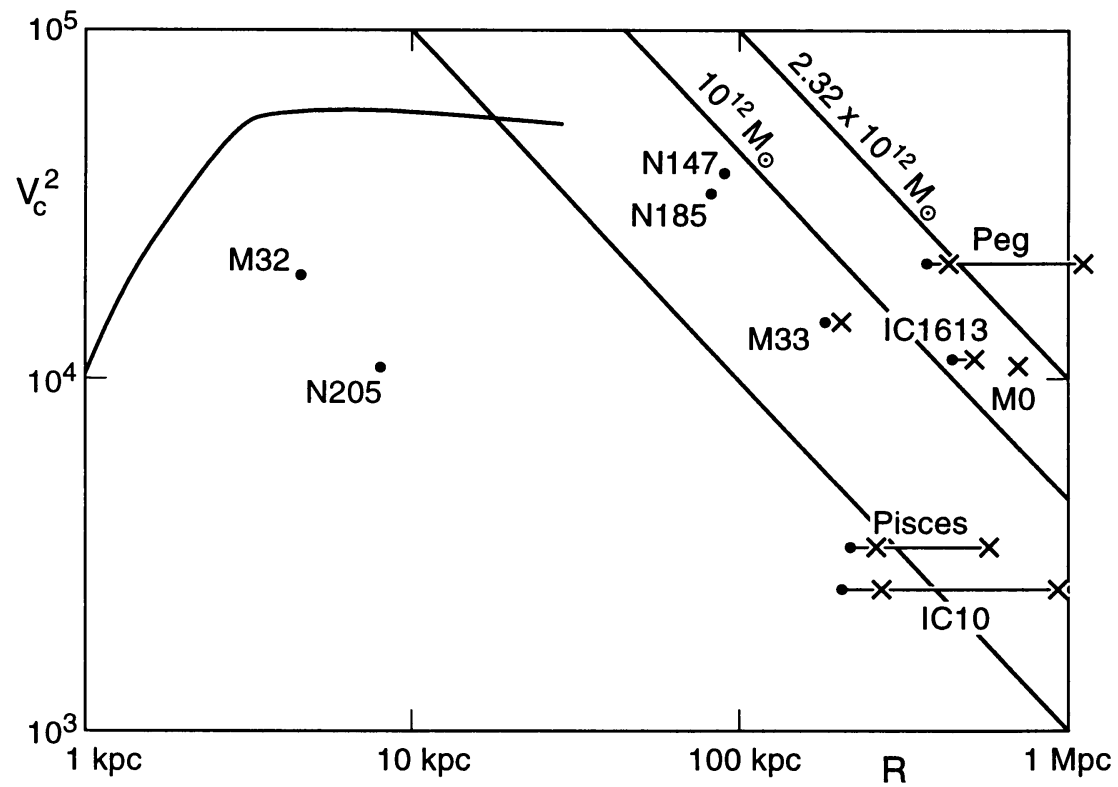

Figure 3. $\quad \log V_{c}^{2}-\log R$ plot for the circular velocity of M31 from its rotation curve and its satellites. Dots denote projected distances; $\times$ denotes a true distance from M31. Pegasus, Pisces \& IC 10 have redetermined distances much smaller than old estimates. M0 represents the Milky Way Galaxy.

If further we assume the line of sight is typical so that averaging over the satellites $\left\langle\dot{\mathbf{r}}^{2}\right\rangle=3\left\langle V_{\ell}^{2}\right\rangle$ where $V_{\ell}$ is the velocity in our line of sight, but reduced to Andromeda's frame, we find

$$
3\left\langle V_{\ell}^{2}\right\rangle=\left\langle V_{c}^{2}\right\rangle .
$$

In Fig. 3 we plot $\log V_{c}^{2}$ against $\log r$ both for the rotation curve and the satellites but for the latter we plot $\log 3 V_{\ell}^{2}$ and $\log R$ ( $R$ is the projected distance to M31). When an object's true distance from M31 is reliably estimated we join that point to a cross at the true distance.

The Local Group members that are within $60^{\circ}$ of M31 on the sky and are closer to it than they are to the Milky Way we assign to the Andromeda group, although the last two EGB and WLM may not be particularly associated with M31 rather than the Local Group as a whole.

From 11 satellites one might hope to get a value of $\left\langle V_{c}^{2}\right\rangle^{1 / 2}$ in rough agreement with the amplitude of the rotation curve but this is not fulfilled $\left\langle V_{c}^{2}\right\rangle^{1 / 2}=$ $141 \mathrm{~km} \mathrm{~s}^{-1}$ significantly below M. Roberts' result, $220 \mathrm{~km} \mathrm{~s}^{-1}$ (Roberts \& Rots 1973). It could be that the outer satellites of M31 have a negative $\frac{1}{2}(d / d t)^{2}\left\langle r^{2}\right\rangle$ as they accelerate back toward it for the first time.

From Table 1 we see that most of M31's satellites move away from us in M31's system of rest. Since the positive numbers are on average larger than the 
Table 1. Andromeda Subgroup

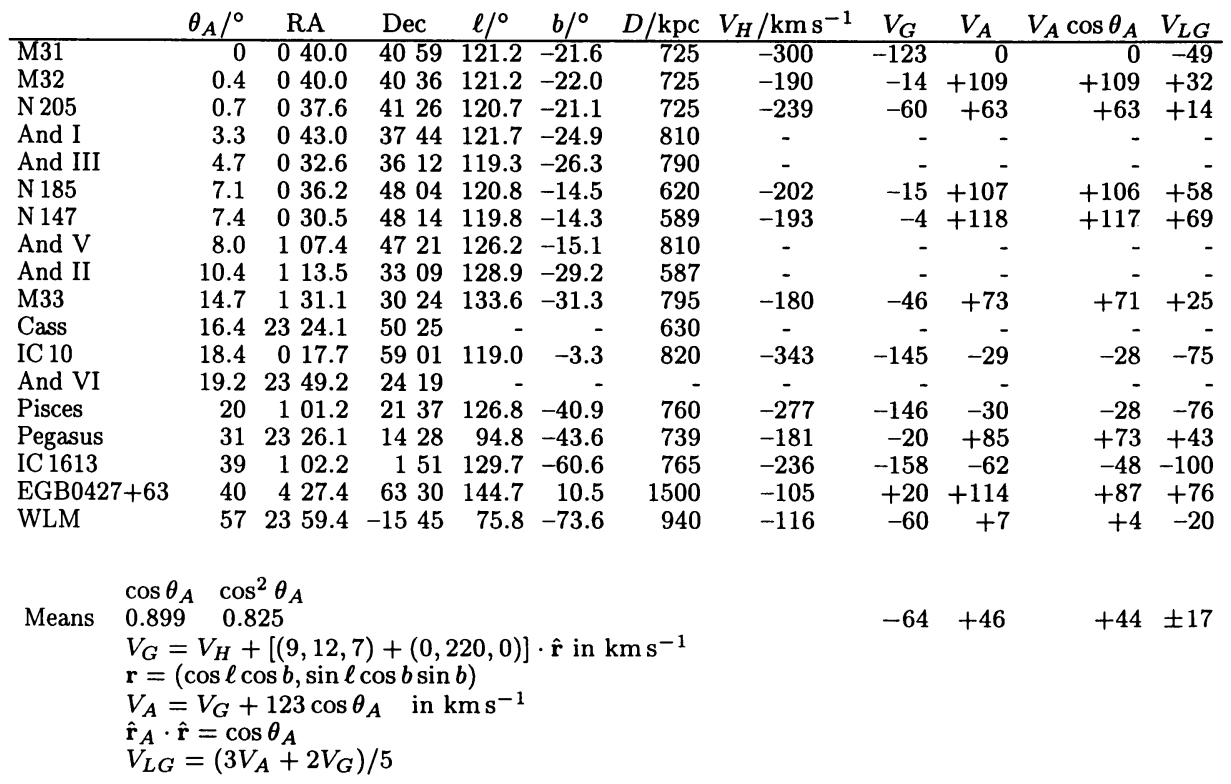

negative ones it seems that M31 moves relative to the mean. If the galaxies, M31 included, were insignificant pimples in a sea of dark matter then that sea could move with the mean velocity of the group, i.e., $72 \mathrm{~km} \mathrm{~s}^{-1}$ toward the Milky Way rather than with the $123 \mathrm{~km} \mathrm{~s}^{-1}$ approach velocity of M31 itself. We determine the mean velocity of the Andromeda group in two ways. Firstly we find that system of rest in which the mean velocities along the lines of sight from the sun is zero. This gives the value $-72 \pm 18 \mathrm{~km} \mathrm{~s}^{-1}$ quoted above. Alternatively we calculate $\left\langle V_{A} \cos \theta_{A}\right\rangle /\left\langle\cos ^{2} \theta_{A}\right\rangle$ which gives $(44 \pm 17) / 0.825=+53 \pm 21 \mathrm{~km} \mathrm{~s}^{-1}$ in M31's frame and then add the velocity of M31, viz -123 to get $-70 \pm 21$, the good agreement from the same data is hardly surprising. We use the earlier value henceforth. M31's velocity of -123 is 2.8 standard deviations from the mean, which would occur in less than $1 \%$ of trials if the true distribution were Gaussian. However, the distribution of velocities is not Gaussian and some of the velocities are correlated, e.g., as Van den Bergh (1988) has pointed out NGC 147 and 185 are probably a bound pair so probably should be treated as a single object in doing the statistics. If we omit the last two doubtful satellites, the remaining velocities then split 5 positive to 3 negative and two of the positives M32 and NGC 205 are well within the region in which we know the gravity field is centred on M31 from the observed rotation curve. Thus it remains more likely that the bulk of the dark matter moves with M31 and the observed velocities of the satellites happen to be rather lopsided. M31 will outrun its satellites but as it does so its gravity field is quite capable of reversing their velocities so we shall not see M31 $500 \mathrm{kpc}$ ahead of its satellites in $10^{10}$ years as their current 51 $\mathrm{km} \mathrm{s}^{-1}$ velocity difference might imply. 
Table 2. Milky Way Subgroup

\begin{tabular}{|c|c|c|c|c|c|c|c|c|c|}
\hline & $\theta_{A} /^{\circ}$ & RA & Dec & $\ell /{ }^{\circ}$ & $b /{ }^{\circ}$ & $D / \mathrm{kpc}$ & $V_{H} / \mathrm{km} \mathrm{s}^{-1}$ & $V_{G}$ & $V_{G} \cos \theta_{A}$ \\
\hline M0 & 119 & 1742.4 & -2855 & 0 & 0 & 8.5 & -9 & 0 & 0 \\
\hline Sagittarius & 107 & 1851.9 & -3030 & 5.7 & -14.1 & 24 & 140 & 170 & -51 \\
\hline LMC & 122 & 524.0 & -6948 & 280.5 & -32.9 & 49 & 270 & 76 & -40 \\
\hline SMC & 114 & 051.0 & -7306 & 302.8 & -44.3 & 58 & 163 & 23 & -10 \\
\hline Ursa Minor & 68 & 1508.2 & 6723 & 105.0 & +44.8 & 69 & -250 & -88 & -33 \\
\hline Draco & 65 & 1719.2 & 5758 & 86.4 & +34.7 & 76 & -289 & -94 & -39 \\
\hline Sculptor & 75 & 057.6 & -3358 & 287.8 & -83.2 & 78 & 107 & 75 & +19 \\
\hline Carina & 121 & 640.4 & -5055 & 260.1 & -22.2 & 87 & 223 & 7 & -3 \\
\hline Sextans & 128 & 1010.6 & -124 & 243.6 & +42.3 & 90 & 224 & 73 & -45 \\
\hline Fornax & 80 & 237.8 & -3444 & 237.3 & -65.7 & 131 & 53 & -34 & -6 \\
\hline Leo II & 113 & 1110.8 & +2226 & 220.1 & +67.2 & 230 & 76 & 24 & -9 \\
\hline Leo I & 116 & 1005.8 & +1233 & 226.0 & +49.1 & 270 & 285 & 177 & -76 \\
\hline Phoenix & 87 & 149.0 & -4442 & 272.2 & -68.9 & 390 & 56 & -32 & -2 \\
\hline N6822 & 89 & 1942.0 & -1456 & 25.3 & -18.4 & 540 & -49 & +49 & +1 \\
\hline & os & & & & & & & & \\
\hline Mean & 0.354 & & & & & & & $30 \pm 22$ & $-21 \pm 7$ \\
\hline
\end{tabular}

\section{The Milky Way Subgroup}

From Fig. 2 we saw that there is a significant concentration of galaxies around the Milky Way (M0). Whereas some of this is undoubtedly due to observational selection, many of the members of this subgroup would have been seen wherever they were within the Local Group so the Milky Way's subgroup is not created by such selection. Table 2 lists the galaxies within $550 \mathrm{kpc}$ of the Centre of the Galaxy. Again, as for the Andromeda group, the last two galaxies may or may not be members of the group but their inclusion or exclusion hardly changes the statistics and has no bearing on the main conclusions. Since the main axis of the Local Group lies along the line between the Milky Way and M31 we still list the angle $\theta_{A}$ and $V_{G} \cos \theta_{A}$. To work out the subgroup's velocity toward M31 we first calculate $\left\langle V_{G} \cos \theta_{A}\right\rangle=-21 \pm 7$; dividing by $\left\langle\cos ^{2} \theta_{A}\right\rangle=0.354$ we find a velocity of $V_{0}=-59 \pm 20 \mathrm{~km} \mathrm{~s}^{-1}$ for the Milky Way's subgroup along the line to M31 in the Milky Way's system of rest. Again the major galaxy seems to be moving with respect to the mean with almost three standard deviations of the mean. The mean velocities of the two groups along their line of separation are remarkably similar. In the Milky Way's system of rest the motions along the line to M31 are

$$
V_{0}=-59 \pm 20 \mathrm{~km} \mathrm{~s}^{-1} \quad V_{A}=-72 \pm 18 \mathrm{~km} \mathrm{~s}^{-1}
$$

which gives a relative velocity of the two means along that line of $-13 \pm 27$ $\mathrm{km} \mathrm{s}^{-1}$. However, the picture in which there is one great bath of dark matter does not explain why there are two prominent subgroups centred on the major galaxies. One could be led to consider the two dark matter pools close to the major galaxies and, although the picture in which the major galaxies move with respect to their local pools leads to much less dark matter because the pools have a much lower approach speed, nevertheless we shall find that the very slow approach velocity is not in as good agreement with data from far out satellites as the faster approach speed of the major galaxies, so we adopt the picture with the dark matter moving with them. 


\section{Dumb-Bell Dynamics}

As long ago as 1959 F.D. Kahn \& L. Woltjer wrote the basic paper on Local Group Dynamics and I pay tribute to my friend Franz Kahn who died earlier this year by quoting their main results here.

They used a velocity of approach of M31 and M0 of $125 \mathrm{~km} \mathrm{~s}^{-1}$ and decided that the period must be less than 15 billion years. They deduced a MINIMUM effective mass [which I deduce to be $M_{A}^{3} /\left(M_{A}+M_{G}\right)^{2}$ ] to be $1.8 .10^{12} \mathrm{M}_{\odot}$. This mass was so much larger than the masses of galaxies believed at that time that they had to find extra mass. We would now call it dark matter but they suggested gas at a density of $1.6 \times 10^{-28} \mathrm{gm} \mathrm{cm}^{-3}$ pervading the Local Group at a temperature of $5 \times 10^{5} \mathrm{~K}$. They pointed out that a straight line orbit minimised the mass required with all other orbits requiring yet more. The works of Sinclair Smith and Zwicky on the large clusters Coma \& Virgo had already raised this problem afar but Kahn \& Woltjer brought it to our own back door!

The equation which governs the radial motion of two masses is

$$
\ddot{r}=-G M / r^{2}
$$

where $M=M_{A}+M_{G}$ is the sum of the masses. It is readily solved parametrically in terms of the cycloidal angle $\eta$ familiar from cosmology. It is interesting to notice that a distant member of the Local Group will feel the gravity of the Milky Way and Andromeda pulling in almost the same direction so the radius vector to it will also obey equation (2) with the same mass $M$. It also will share the initial condition $r=0$ at $t=0$ the Big Bang. If one eliminates the other initial condition (the energy) and $\eta$ from the equations for $r(\eta), v(\eta)$ and $t(\eta)$ one finds that $\left(G M / r^{3}\right)^{1 / 2} t$ is a definite function of $(v t / r)$ given by the curve of Fig. 4. For $v t / r<\frac{1}{2}$ this curve is well represented by the straight line

$$
\left(\frac{G M}{r^{3}}\right)^{1 / 2} t+0.85 \frac{v t}{r}=1.11 .
$$

If we therefore plot $r^{-3 / 2}$ against $v / r$ both for M31 which gives $r=0.725 \mathrm{Mpc}$, $v=-1.23 \times 100 \mathrm{~km} \mathrm{~s}^{-1}$ and for the $r$ 's and $v$ 's of distant satellites of the Local Group where those (but not M31's) must be measured from the barycentre of the Local Group, then we can expect an almost straight line whose gradient will be $0.85(G M)^{-1 / 2}$ and whose intercept at the stand-still sphere $v=0, r=r_{0}$ will give

$$
r_{0}^{-3 / 2}=\frac{1.11}{(G M)^{1 / 2} t} .
$$

Thus we can find both the mass of the Local Group and the time $t$ since the Big Bang - i.e., the true age of the Universe from such Local Group studies. These points I made clearly in my 1981 article (Lynden-Bell 1981) but the distances to far out members of the Local Group were then very poor and even now seem to be continually changing as the observations improve. While absolute distances are important - they set the whole scale and indeed if $r, v, M, t$ satisfy equation (3), or the exact equation to which it is an approximation, then one sees that $\lambda r, v, \lambda M, \lambda t$ will do so as well, so a rescaling of all Local Group distances will rescale the mass of the Local Group and the age of the Universe by exactly the 


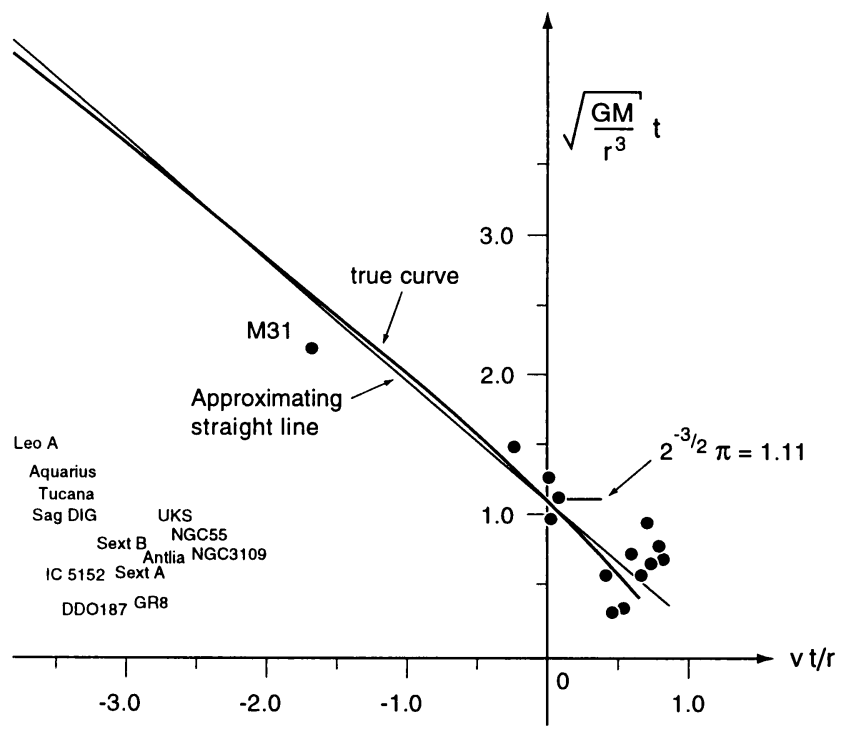

Figure 4. $\quad \sqrt{G M} t r^{-3 / 2}$ against $v t / r$ for straight line motion of two bodies. The straight line is a good approximation in the range $-3<\omega t<0.5$. The points plotted are $\frac{4}{3} r^{-3 / 2}$ against $v / r$ with $v$ in hundreds of $\mathrm{km} \mathrm{s}^{-1}$ and $r$ in Mpc.

same factor. In practice we plot $\frac{4}{3} r^{-3 / 2}$ with $r$ in Mpc against $v / r$ with $v$ in hundreds of $\mathrm{km} \mathrm{s}^{-1}$ because with the choice of $\frac{4}{3}$ as gradient the observational points of Table 3 fall close to the theoretical curve of Fig. 4.

The intercept at $v=0$ is quite well defined by the 4 central points for Leo A, Aquarius, Tucana and Sag DIG. These give $\frac{4}{3} r_{0}^{-3 / 2}=1.11(1 \pm .1)$ and hence

$$
(G M)^{1 / 2} t=\frac{4}{3}(1 \pm .1) .
$$

If we accept the fit in Fig. 4 with no further rescaling of the gradient to fit M31 exactly then from that gradient

$$
M=\left(\frac{4}{3}\right)^{2} 2.32 \times 10^{12}=4.1 \times 10^{12} M_{\odot}
$$

which gives

$$
t=(1 \pm 0.1) \times 10^{10} \mathrm{yr} .
$$

It is perhaps natural to ask why we do not demand that M31 lie exactly on the curve. Had we done that we would have got the unacceptable result

$$
t=0.73 \times 10^{10} \mathrm{yr} .
$$

The trouble is that the stand-still sphere appears too close in. M31 has already turned around so must have reversed earlier and therefore nearer than the standstill sphere that we now observe. To accelerate M31 back in so short a distance 
Table 3. Unattached

\begin{tabular}{|c|c|c|c|c|c|c|c|c|c|c|c|}
\hline & $\theta_{A} /^{\circ}$ & $\mathrm{RA}$ & Dec & $\ell /{ }^{\circ}$ & $b /{ }^{\circ}$ & $D / \mathrm{kpc}$ & $V_{H} / \mathrm{km} \mathrm{s}^{-1}$ & $V_{G}$ & $V_{G} \cos \theta_{A}$ & $V_{L G}$ & $r / \mathrm{Mpc}$ \\
\hline N3109 & 144 & 1000.8 & -2555 & 262.1 & +23.1 & 1260 & 403 & 193 & -156 & 134 & 1631 \\
\hline Sextans A & 124 & 1008.6 & -0428 & 246.2 & +39.9 & 1450 & 325 & 164 & -91 & 123 & 1762 \\
\hline Sextans B & 131 & 957.4 & +0534 & 233.2 & +43.8 & 1300 & 301 & 168 & -110 & 119 & 1657 \\
\hline Leo A & 99 & 956.5 & +3059 & 196.9 & +52.4 & 692 & 26 & -10 & 2 & -21 & 871 \\
\hline Sag DIG & 93 & 1927.9 & -1747 & 21.1 & -16.5 & 1150 & -79 & 6 & 0 & +2 & 1247 \\
\hline Antlia & 145 & 1001.8 & -2705 & 263.1 & +22.3 & 1150 & 361 & 150 & -123 & 90 & 1534 \\
\hline Tucana & 108 & 2238.5 & -6441 & 322.9 & -47.4 & 900 & $\dagger(130)$ & (36) & $(-11)$ & (13) & 1113 \\
\hline IC 5152 & 99 & 2159.6 & -5132 & 343.9 & -50.2 & 1600 & 121 & 81 & -13 & 70 & 1718 \\
\hline UKS2323- & 76 & 2323.8 & -3240 & 11.9 & -70.9 & 1300 & 62 & 76 & +18 & 94 & 1264 \\
\hline Aquarius & 77 & 2044.2 & -1301 & 34.1 & -31.4 & 1030 & -131 & 18 & 4 & -1 & 1018 \\
\hline N 55 & 81 & - & & 332.9 & -75.7 & 1600 & 129 & 109 & 17 & 120 & 1507 \\
\hline GR8 & 124 & 1256.2 & +1429 & 310.8 & +77.0 & 2200 & 216 & 185 & -103 & 144 & 2470 \\
\hline DO 187 & 112 & - & & 25.6 & +70.5 & 2400 & 153 & 181 & -68 & 125 & 2692 \\
\hline
\end{tabular}

\footnotetext{
${ }^{\dagger}$ The velocity here attributed to Tucana is the $21 \mathrm{~cm}$ velocity of a cloud of hydrogen quite near it in the sky but with no definitive connection to Tucana itself.
}

needs a large $M$ and hence a small $t$. Both ages (5) and (6) are much shorter than those involved in stellar evolution would like. Can we alleviate this? To some extent we can if we follow Prof. Feast in changing the distance scale within the Local Group. This has the added advantage of making the stars in, e.g., the globular clusters further away and hence brighter, heavier and younger. We have used a distance of $725 \mathrm{kpc}$ to M31 whereas Feast gives a distance modules to the LMC of 18.70 and to M31 of 24.70. Thus on his scale M31 is $870 \mathrm{kpc}$ distant, i.e., 1.2 times as far away as we saw earlier, $t$ and $M$ scale with the distance. If we adopt Feast's new distances then our results become:

The time since the Big Bang is

$$
t=(1.2 \pm 0.1) \times 10^{10} \mathrm{yr} .
$$

The Mass of the Local Group is

$$
M=4.9 \pm .810^{12} M_{\odot} .
$$

If this is still considered too short an age we can try to invoke an orbit for M31 that involves some transverse motion but my 1981 analysis shows that this only shortens $t$. One possible further alleviation is to take orbits of eccentricity $e$ for the objects defining the stand-still sphere. Then the time since the Big Bang would be increased by the factor

$$
2\left(\frac{2}{1+e}\right)^{3 / 2}-1=\left\{\begin{array}{cl}
1.3 & \text { for } e=0.8 \\
1.16 & \text { for } e=0.9
\end{array}\right.
$$

There is, however, a limit on how large we can make $e$ since the distribution of the $e$ will give a spread in distance to those defining the stand-still sphere of $\frac{1}{2}(1+e) r_{0}$ and we already estimated this at no more than $0.1 r_{0}$ corresponding to a maximum $e$ of 0.8 . Perhaps the most likely revision of the age will come 


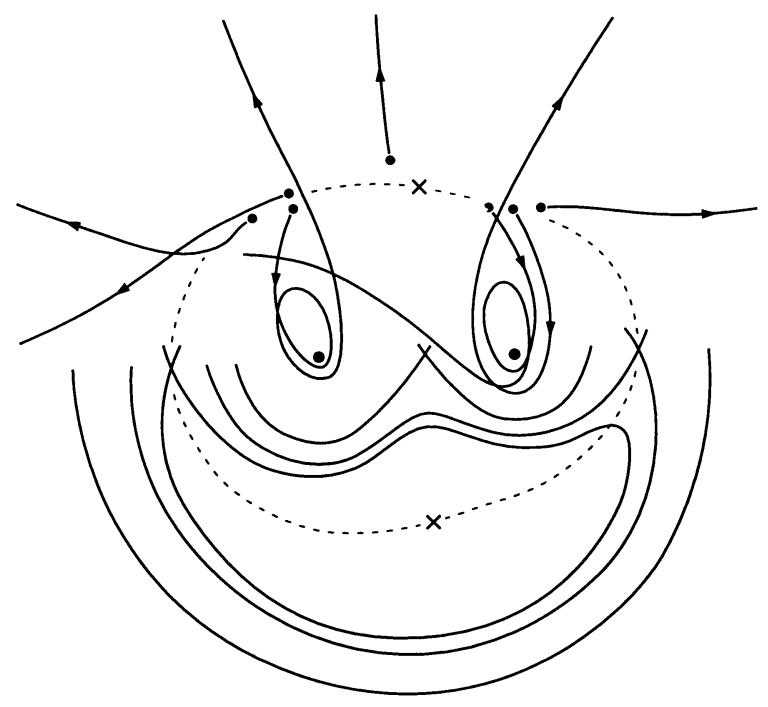

Figure 5. The lower half of this figure plots the critical equipotentials of $-\psi$, the gravitational potential of the Local Group in comoving coordinates. The rampart or ridge line off which the galaxies will fall is the dotted line. The eyes are M31 on the left and the Galaxy on the right. Several orbits starting near the ridge line are plotted in the upper half. The two $\times$ mark the triangular Lagrangian points.

from accurate revision of the relative distances of M31 and the 4 crucial objects Leo A, Aquarius = DDO 210, Tucana and Sag DIG.

Of course it is possible to use all the Local Group not just the far outliers. Some years ago I gave the problem of computing the orbits back to the Big Bang to my then student Mishra (1985). Following his attempt to do this, Peebles $(1989,1990,1994)$ invented a very elegant way of incorporating the initial conditions at the Big Bang via a variational principle. However, such methods have their own problems in that the variational principle is only a stationary one and the true path may not be one of minimum action. Further the broad distribution of dark matter around the central galaxies makes the use of the closer satellites rather uncertain. Different practitioners of the method have found rather discrepant results, cf. Dunn \& LaFlamme (1993, 1995), Schmoldt \& Saha (1998).

I have therefore preferred the very simplistic approach above in which one can see more clearly how the dynamics gives the age and the mass of the Local Group. In Lynden-Bell (1982) I tested the accuracy of equation (3) by computing orbits for small satellites. If one demands that they emerge from the Big Bang with almost no peculiar motion, since that would diverge if extrapolated back, then the forces have to balance initially. Thus the small bodies must emerge at close to the triangular points of Lagrange (Fig. 5) giving an equilateral triangle with M0 and M31. 


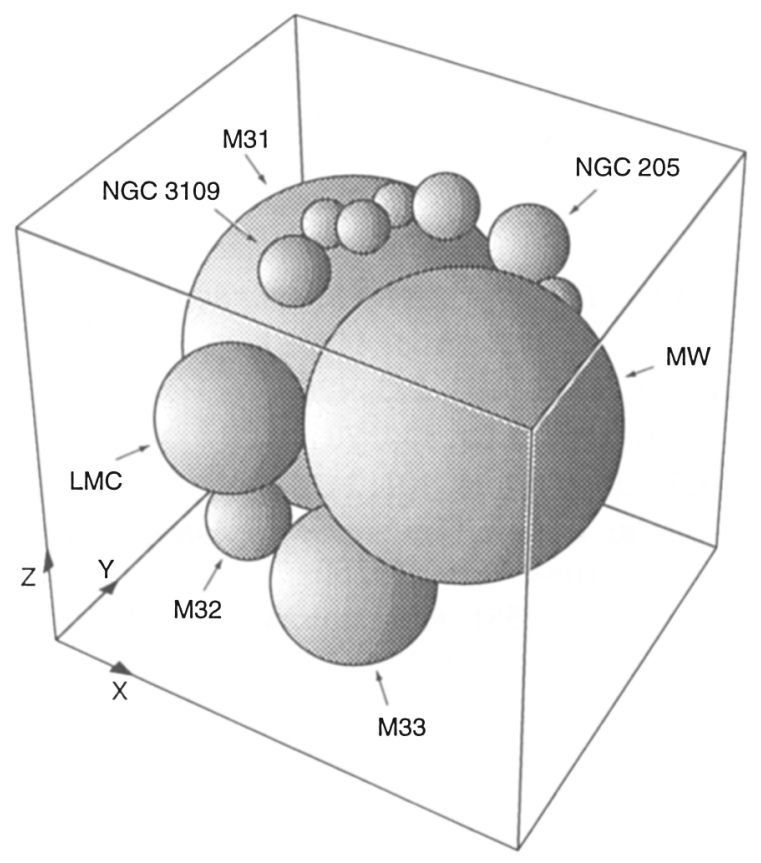

Figure 6. Alan Whiting's picture of a possible initial condition for the Local Group. The smaller galaxies huddle around the Lagrangian ring of M31 and the Milky Way.

These points form a circle about the axis joining the two major galaxies. A remarkable picture of Alan Whiting's (1997) shows a possible initial condition for the Local Group with all the minor galaxies huddled around this circle (Fig. 6).

Other refinements have been tried. In a very fine paper Gott \& Thuan (1978) looked into the generation of the spins of Local Group galaxies pointing out that the primary interaction was the differential tug of a protogalaxy acting as a monopole on the quadrupole moment presented by another. The spins so generated are perpendicular to the line originally joining the galaxies and it was remarked that we are now only $11^{\circ}$ from M31's plane while it is only $21^{\circ}$ off ours. However, their attempt to make the total angular momentum of the Local Group to be zero led to a very large $135^{\circ}$ motion of M31 in the sky which was not in good agreement with an original straight line orbit. Raychaudhury \& I (1989) evaluated the external torques that would have generated some motion in M31 but the orbits found were not such as to change the times estimated by the simple Kahn \& Woltjer straight line orbit.

It is remarkable that the separation of the Milky Way and M31 lies close to the Supergalactic Plane. This flattened structure continues to at least 4000 $\mathrm{km} \mathrm{s}^{-1}$ redshift and Hudson, Smith \& Lacey find the Large Scale Streaming motion continues to a redshift of $12000 \mathrm{~km} \mathrm{~s}^{-1}$. This motion relative to the CMB is close to that of the Local group itself. 
Acknowledgements. I particularly thank M.J. Irwin for his encouragement and for sharing his new results with me. The new data provided at this conference has been used to revise the numbers and so bring the talk up to date.

\section{References}

Dunn, A.M., LaFlamme, R. 1993, MNRAS, 426, 865

Dunn, A.M., LaFlamme, R. 1995, ApJ, 443, L1

Gott, J.R., Thuan, T.X. 1978, ApJ, 223, 426

Kahn, F.D., Woltjer, L. 1959, ApJ, 130, 705

Lynden-Bell, D. 1981, Observatory, 101, 111

Lynden-Bell, D. 1982, in: Vatican Conference on Astrophysical Cosmology, Pontifical Academy Scripta Varia, 48, 85

Lynden-Bell, D., Frenk, C.S. 1981, Observatory, 101, 200

Mishra, R. 1985, MNRAS, 212, 163

Peebles, P.J.E. 1989, ApJ, 344, L53

Peebles, P.J.E. 1990, ApJ, 362, 1

Peebles, P.J.E. 1994, ApJ, 429, 43

Raychaudhury, S., Lynden-Bell, D. 1989, MNRAS, 240, 195

Roberts, M.S., Rots, A.H. 1973, A\&A, 26, 483

Schmoldt, I.M., Saha, P. 1998, Oxford Preprint

Van den Bergh, S. 1998, ApJ, 505, L127

Whiting, A. 1997, Thesis, Cambridge University

\section{Discussion}

Lee: I have obtained a good estimate for the distance to the Aquarius galaxy (DDO 210), showing the results in the Poster (Lee these proceedings). The distance to this galaxy is estimated to be $1030 \mathrm{kpc}$, and the distance of this galaxy from the center of the Local Group is derived to be $930 \mathrm{kpc}$. This result is consistent with your prediction.

Lynden-Bell: That's fine - it will fit well with WLM.

Zabludoff: With respect to the question of why the fractional contribution of gas to the mass of the Local Group appears to be less than that of rich clusters, recent work suggests indirectly that the gas fractions may not in fact be different. Given the observed correlation between X-ray luminosity and velocity dispersion for poor groups and rich clusters, we would not expect the shallow Local Group potential to heat gas to X-ray detectable levels. There may instead be cold or warm gas in the Local Group that could be detected in the extreme UV or in HI (including the high velocity, cold clouds discussed by Blitz and Spergel).

Lynden-Bell: I am delighted and amazed to hear that, but I shall await with anticipation a definitive demonstration that the gas is really there! 